\title{
La prévisualisation 3D et ses apports vidéoludiques : comment jouer à faire un film?
}

\section{Benoit Melançon}

\section{(2) OpenEdition}

1 Journals

Édition électronique

URL : http://journals.openedition.org/sdj/694

DOI : $10.4000 /$ sdj. 694

ISSN : 2269-2657

\section{Éditeur}

Laboratoire EXPERICE - Centre de Recherche Interuniversitaire Expérience Ressources Culturelles Education

\section{Référence électronique}

Benoit Melançon, «La prévisualisation 3D et ses apports vidéoludiques : comment jouer à faire un film ?», Sciences du jeu [En ligne], 6 | 2016, mis en ligne le 16 octobre 2016, consulté le 19 avril 2019. URL : http://journals.openedition.org/sdj/694 ; DOI : 10.4000/sdj.694

Ce document a été généré automatiquement le 19 avril 2019.

Tous droits réservés 


\title{
La prévisualisation 3D et ses apports vidéoludiques : comment jouer à faire un film?
}

\author{
Benoit Melançon
}

1 Des différents liens existant entre l'art et le jeu, le détournement de dispositifs vidéoludiques à des fins de création artistique demeure l'un des plus fascinants: de l'utilisation de consoles portables Game Boy pour créer des pièces musicales (Driscoll et Diaz, 2009) jusqu'à des effets de time-lapse représentant les paysages en mouvement à l'intérieur d'un jeu vidéo (Powers, 2011), les exemples d'œuvres témoignant de l'ingéniosité d'artistes bricoleurs sont aussi nombreux que variés. À l'instar de la musique et de la photographie, le cinéma fait lui aussi partie des arts ciblés par de tels détournements.

2 Réaliser un film à l'aide d'un jeu vidéo n'est pas une idée nouvelle : depuis le milieu des années 1990, le terme «machinima » désigne des récits créés au sein d'environnements vidéoludiques, un phénomène aujourd'hui substantiellement documenté. À l'origine, les créateurs de ces courts métrages étaient des joueurs possédant les connaissances suffisantes pour détourner momentanément un jeu vidéo de son principal usage ludique afin d'élaborer une mise en scène inspirée du cinéma, mise en scène qui était par la suite traduite en images. Plus récemment, l'apparition d'outils destinés spécifiquement à la création de machinimas est venue non seulement démocratiser cette nouvelle discipline narrative, mais également susciter de nouveaux questionnements quant aux activités liées à ces dispositifs situés à mi-chemin entre le jeu vidéo et le cinéma.

3 Tout en offrant des pistes visant à baliser l'emploi de tels logiciels permettant de créer des œuvres cinématographiques dans des espaces virtuels, le présent texte examinera comment ces nouveaux systèmes peuvent participer à la préparation d'un tournage véritable par le biais de la construction d'un "pré-film», un brouillon visuel du film à venir, destiné à la fois à guider les techniciens durant la production en milieu réel, et également à aider le réalisateur à visualiser son œuvre d'une manière qui lui paraîtra 
optimale. Cet examen considérera comme point de départ la définition de la pratique du jeu telle que proposée par Bernard Suits :

Pratiquer un jeu est une tentative pour atteindre un but particulier (but préludique), en utilisant les seuls moyens autorisés par les règles (moyen ludique), où les règles interdisent l'usage de moyens plus efficaces au profit de moyens qui le sont moins (règles constitutives), et où de telles règles sont simplement acceptées parce qu'elles rendent possible une telle activité (attitude ludique) (Suits, 2006, p. 190, traduction proposée par Guttman, 2006, p. 22).

L'hypothèse proposée est qu'un jeu vidéo permettant de réaliser des esquisses de récits filmiques peut être considéré non plus tout à fait comme un jeu, mais plutôt comme semblable à un jouet, « un objet en volume dont [on] use librement, sans référence à des règles du jeu ou un principe d'usage d'une autre nature " (Brougère 1992, p. 38). Cette proposition implique l'examen des transformations s'appliquant alors audit jeu (les buts, les moyens et les règles du jeu initial) et à ses utilisateurs (le statut de joueur et l'attitude ludique). D'une manière plus large, elle considère également la part grandissante de la prévisualisation numérique dans le processus de fabrication d'un film, et ses conséquences sur l'acte créatif de l'auteur-réalisateur.

\section{La prévisualisation numérique}

5 Conceptualiser de manière visuelle le film que l'on désire réaliser constitue un aspect particulier de la préproduction cinématographique désigné sous le nom (quelque peu ambigu) de prévisualisation :

On désigne sous le terme de prévisualisation l'ensemble des outils permettant à un réalisateur en cinéma de prévoir, de manière visuelle, le résultat d'un tournage à venir. Le scénarimage [storyboard] en est un exemple. Plus communément, on appelle aujourd'hui prévisualisation ce qui porte en fait le nom d'animatique, c'està-dire une séquence d'images en mouvement qui simule, à la manière d'un brouillon, la version définitive d'un plan ou d'une scène de film telle qu'ils apparaîtront à l'écran (Melançon, 2011, p.1).

Depuis les débuts du cinéma, des réalisateurs tels que Méliès, Murnau et Griffith ont eu recours au scénarimage durant la phase préparatoire de leur film (Peeters, Faton, et De Pierpont, 1992). Par la suite, l'usage de cet outil apparenté à la bande dessinée s'est généralisé, alors que les dessinateurs de storyboard participèrent à la conception de longs métrages, mais également de publicités télévisuelles et de cinématiques de jeux vidéo (Figure 1). 
Figure 1
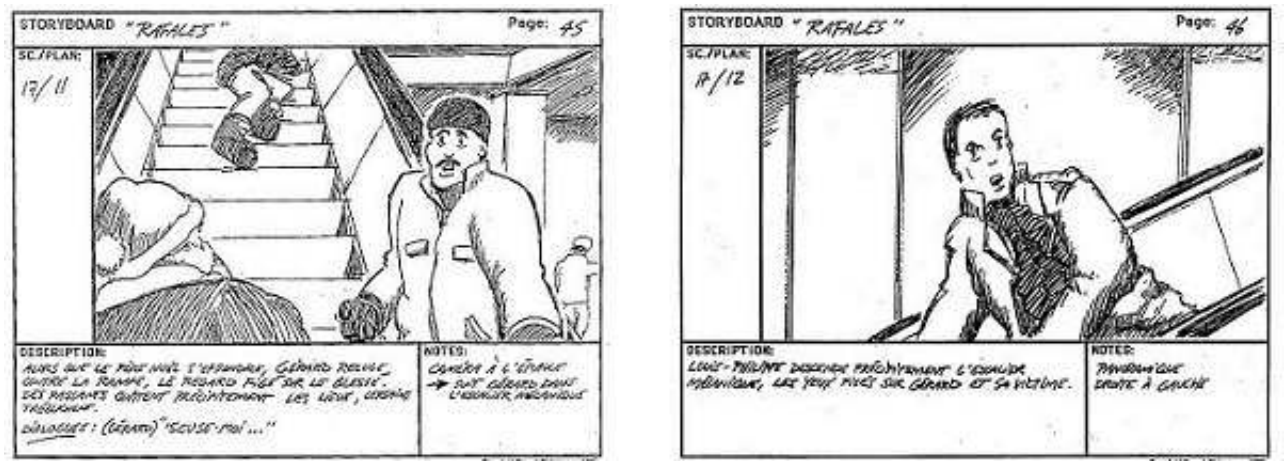

Exemple d'un scénarimage tiré du long métrage Rafales (André Melançon, 1990). Image libre de droits.

7 D'origine plus récente, la prévisualisation dite numérique est quant à elle le plus souvent associée à un certain type de film : la superproduction hollywoodienne impliquant une part importante d'effets visuels, dont le coût élevé du tournage et l'aspect synthétique des trucages (qui les rend invisibles aux techniciens sur le plateau) justifient cette préparation minutieuse. D'une manière similaire, une telle prévisualisation est privilégiée par un type spécifique de réalisateur, puisque certains metteurs en scène vont voir dans ces animatiques parfois très peaufinées le risque de perdre une part de spontanéité lors du tournage proprement dit. En effet, à la différence du scénarimage que l'on peut comparer à une "carte routière indiquant la direction à suivre" lors du tournage (Bouzereau, 1999, ma traduction), l'animatique 3D peut simuler avec exactitude le film à venir en précisant le rythme de la mise en scène et du montage, la justesse des raccords, les mouvements de caméra, le choix de la lentille, la disposition et les dimensions des décors, la gestuelle des comédiens, etc. Cette forme de prévisualisation déplace ainsi en amont du calendrier de production une part importante de la mise en forme de l'œuvre filmique, tout en excluant de cette même mise en forme le cercle traditionnel des artistes et des techniciens présents sur le plateau (Figure 2). 
Figure 2

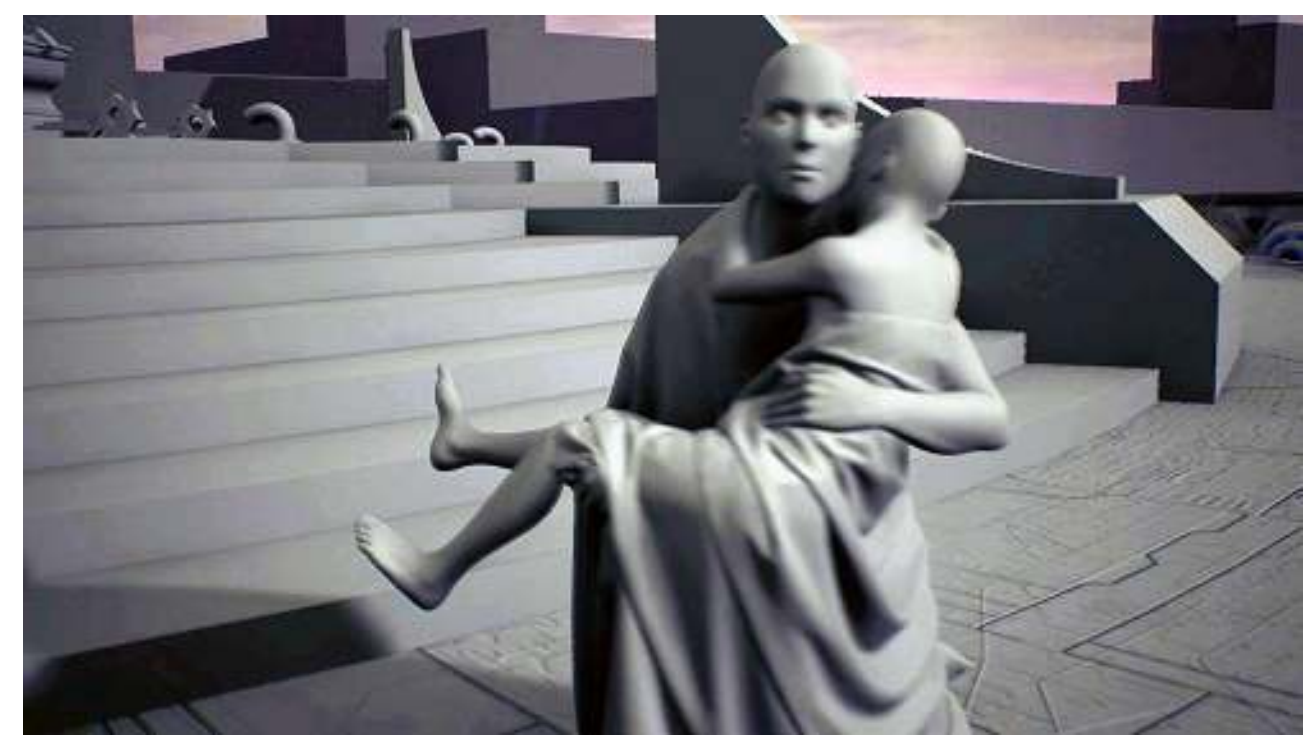

Image extraite de l'animatique 3D du court métrage Monuments (Dave Hawey, 2016). Image libre de droits.

8 Par souci de compatibilité, les logiciels d'infographie 3D utilisés pour la prévisualisation d'un film donné sont habituellement les mêmes que ceux servant à créer par la suite ses effets visuels. En raison de la facture nécessairement photoréaliste desdits effets, ces logiciels favorisent la précision et le détail des objets virtuels, ou « modèles 3D », ainsi que la reproduction fidèle des phénomènes lumineux observables dans le monde réel : en contrepartie, de telles interfaces apparaissent relativement complexes aux yeux d'usagers néophytes, et il n'est pas rare de devoir patienter plusieurs jours avant que des images finales, fruits d'intenses calculs informatiques, puissent enfin être visionnées. Quoique des compromis soient possibles lorsqu'il s'agit de réaliser des animatiques moins esthétiquement achevés que les images visibles sur un écran de cinéma, la prévisualisation numérique demeure une tâche beaucoup plus exigeante et spécialisée que celle du scénarimage, et par conséquent elle se voit presque toujours confiée à des techniciens expérimentés, voire à des entreprises spécialisées dans ce domaine.

assiste cependant depuis quelques années à l'émergence de nouvelles pratiques en prévisualisation qui font appel non plus aux logiciels d'infographie 3D, mais plutôt aux engins de jeu vidéo [game engines]. Ces stratégies comportent deux principaux avantages par rapport à la méthode infographique issue des effets visuels : l'utilisation d'interfaces plus conviviales, et la possibilité de générer en temps réel des images synthétiques de facture presque équivalente à celles du film complété. Le résultat est de permettre à des non-spécialistes (tels que les réalisateurs) de créer rapidement par euxmêmes des animatiques de manière interactive, tout en conservant une qualité visuelle adéquate.

Bien que de telles expérimentations apparaissent suffisamment prometteuses pour susciter une réflexion quant à l'emploi des jeux vidéo dans la préparation visuelle d'un film, insistons ici sur le fait que ces activités demeurent pour l'instant marginales, le plus souvent confinées aux laboratoires de certaines compagnies-phares de l'industrie cinématographique: mentionnons à cet effet le laboratoire ILMxLAB, une initiative de recherche en " narration immersive » (Rubin , 2016) émanant du célèbre studio d'effets 
visuels ILM, et dont le logiciel de prévisualisation Zviz découle des engins de jeux développés par l'ancien éditeur de jeux LucasArts (Bell, 2013). Ce système innovateur n'étant pas disponible au grand public, nous nous tournons aux fins de notre analyse vers un logiciel de nature similaire dont les pratiques connues pourront alimenter notre réflexion.

\section{Un double dispositif}

11 Le présent article examine le cas d'un outil aux origines vidéoludiques utilisable dans un contexte de prévisualisation et propice à exhiber des caractéristiques associées au jouet. Ce dispositif est double : la première composante en est Team Fortress 2 (TF2), un jeu de tir à la première personne multijoueur en ligne créé par l'éditeur de jeux vidéo Valve Software en 2007, et dont le style visuel de type cartoon est accentué par des personnages caricaturaux et des dialogues à saveur humoristique (Figure 3).

Figure $3 \mathrm{~A}$

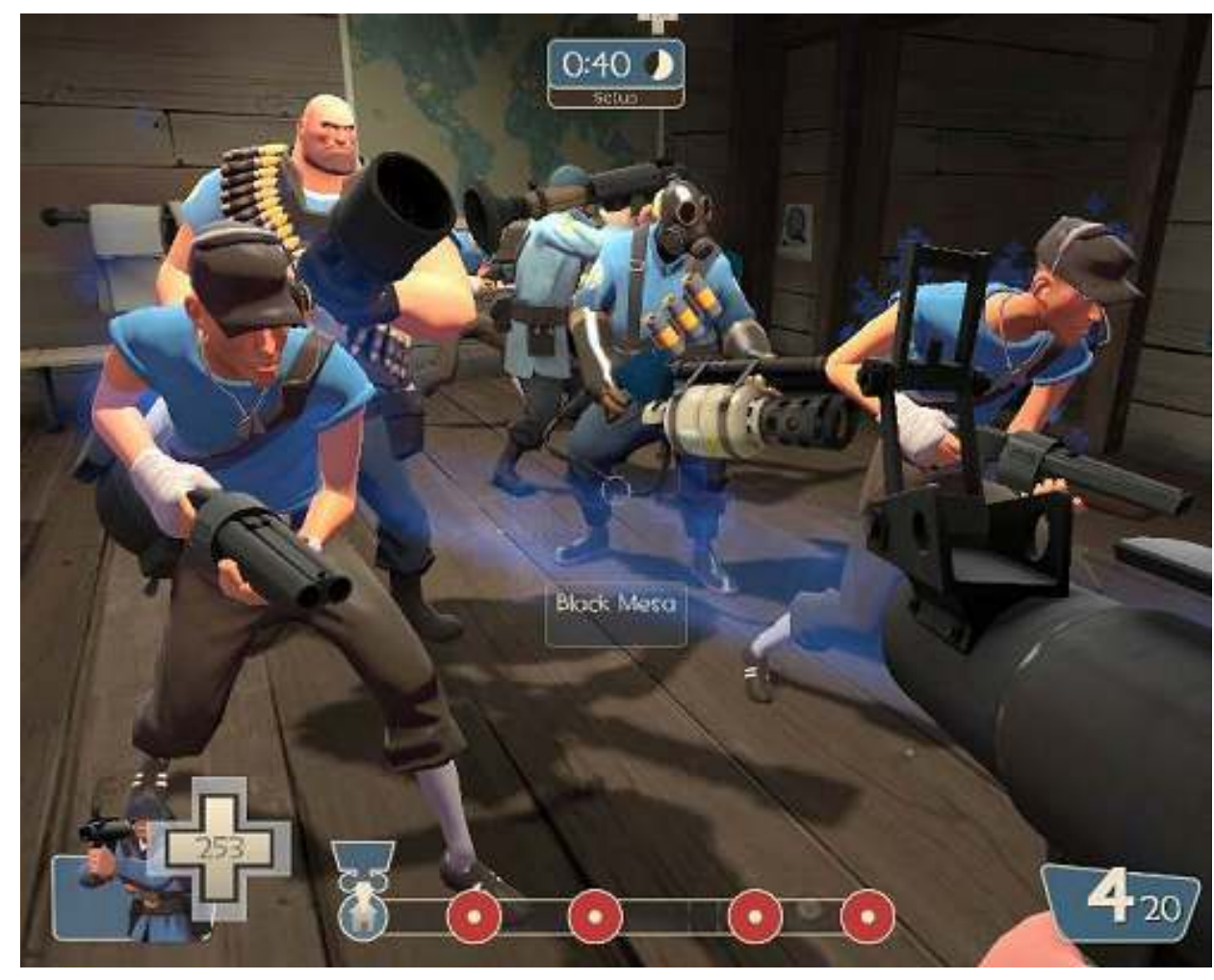

Exemple de l'interface de Team Fortress 2 (Valve Software, 2007). Image libre de droits. 


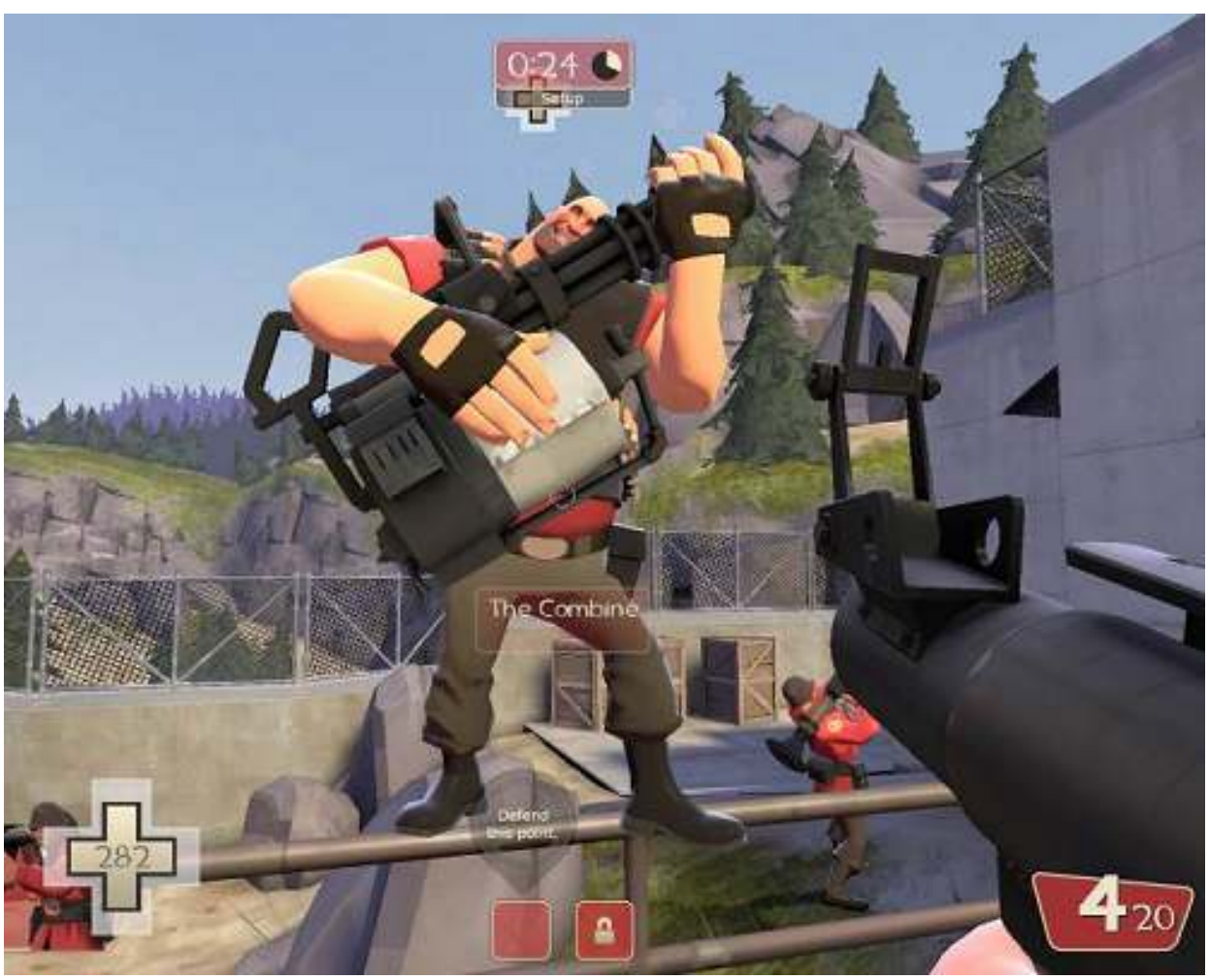

Autre exemple de l'interface de Team Fortress 2 (Valve Software, 2007). Image libre de droits.

Depuis quelques années, la manipulation conventionnelle de ce jeu peut être transformée par une seconde application dénommée Source Filmmaker (SFM), un logiciel de création filmique fonctionnant parallèlement au jeu existant, et qui transforme l'espace diégétique de ce dernier en un plateau de tournage virtuel où les personnages-avatars deviennent des marionnettes pouvant être mis en scène par l'usager afin de progressivement bâtir un récit filmique.

Le binôme SFM/TF2 ne propose donc pas à priori la création ex nihilo d'un univers diégétique, mais plutôt une remédiation des ressources d'un jeu vidéo : tout en offrant une interface et des fonctionnalités propres aux outils de création et de manipulation cinématographiques (telles qu'une ligne de temps [timeline] commune aux logiciels de montage), son principe de fonctionnement est d'accéder différemment au jeu existant, à la manière d'un programmeur qui peut en manipuler des composantes normalement inaccessibles au joueur. Par exemple, en lieu et place des mouvements préprogrammés d'un avatar dans le jeu, le metteur en scène peut modifier le comportement dudit personnage afin de lui faire accomplir une action de son choix (figure 4), et ce par le biais d'une interface similaire à celles utilisées par les studios d'animation tels que Pixar dans la fabrication de films comme Toy Story ou Finding Nemo. 


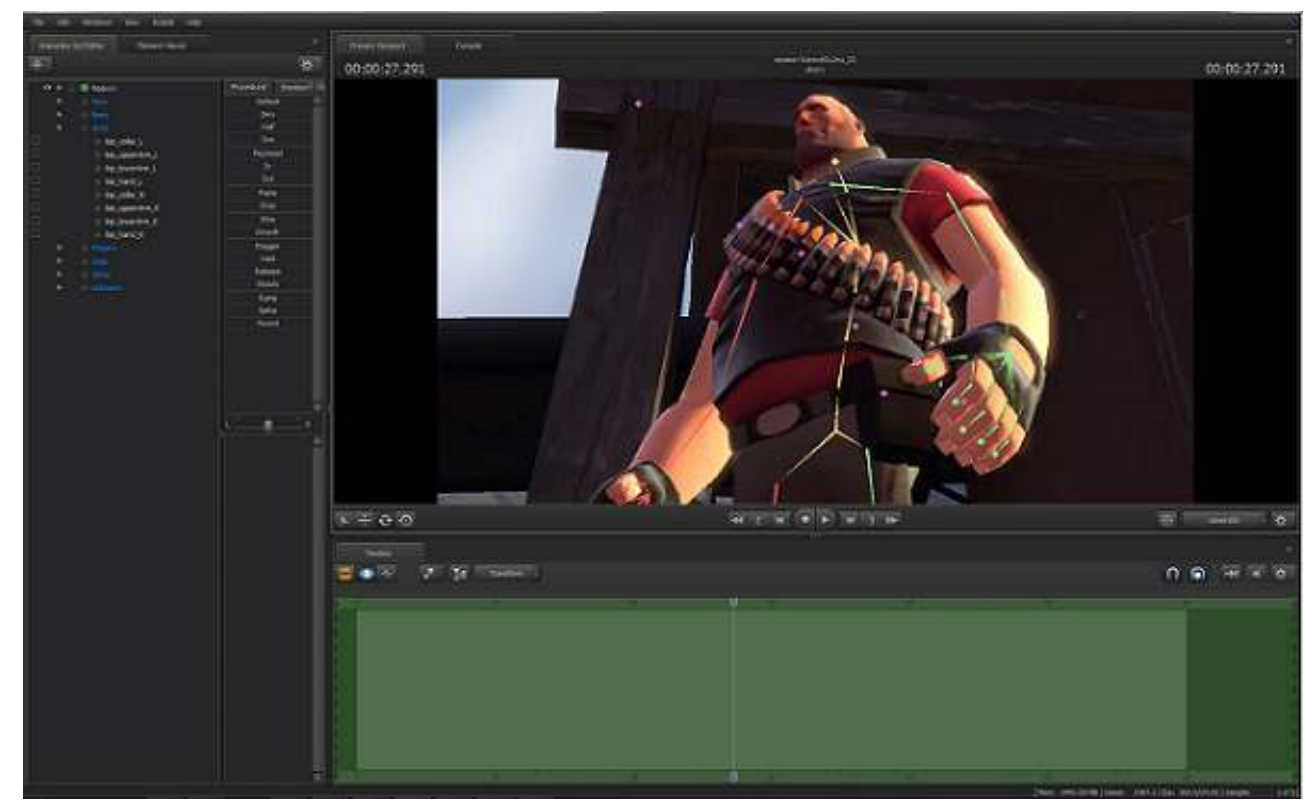

L'armature [rig] d'un avatar de TF2 telle qu'apparaissant dans l'interface de Source Filmmaker. Les phalanges des différents doigts (représentés en vert) peuvent être manipulées par le biais de la souris et du curseur pour enregistrer différentes poses-clés dans le temps qui déboucheront éventuellement sur l'animation d'un mouvement désiré de la main. Image libre de droits.

Parce que l'interface de SFM est appliquée de manière semi-transparente sur celle de TF2, et que sa manipulation dépend en partie de celle de ce dernier, son usager doit savoir jouer à TF2. Contrairement à l'interface originale du jeu qui ainsi perdure, le contexte rattaché à la pratique de TF2 est quant à lui radicalement transformé : le but du jeu (triompher de l'équipe adverse) ainsi que les règles qui encadrent la poursuite de ce but (accomplir cette action dans un temps donné sans que son avatar ne perde la vie) s'effacent au profit d'un nouvel objectif, celui de créer un film prenant place dans l'environnement ludique. À la différence de celui du jeu original, il s'agit là d'un but qui est qualifiable (" produire un film qui finit par nous satisfaire ») plutôt que quantifiable ( « remporter une joute par un nombre $\mathrm{X}$ de points »), puisqu'il est possible de réaliser des films esthétiquement parfaits, mais dont le sujet est monotone ou déplaisant.

C'est désormais à l'utilisateur de déterminer subjectivement s'il a atteint les objectifs proposés par le dispositif en réalisant le film qui lui convient: la satisfaction de cet objectif suppose la maîtrise de l'interface, mais également celle du langage cinématographique. La première compétence peut être adressée par le biais de tutoriaux au sein même du logiciel, alors que la seconde est beaucoup plus difficile à acquérir : il s'agit d'apprendre à faire des films, un savoir-faire subtil qui échappe au cadre de l'outil proprement dit.

\section{Le dispositif en tant que jouet}

16 Bien que son utilisateur appréhende l'univers diégétique du dispositif par le biais d'une médiation bidimensionnelle (l'écran de l'ordinateur), SFM/TF2 offre la possibilité de manipuler des objets synthétiques dans un espace virtuel déployé sur trois axes. Sans égaler la qualité tactile du jouet en milieu réel, on s'approche ici de ses propriétés 
volumiques lorsqu'il s'agit de le manipuler dans l'espace, bien que ce dernier demeure virtuel. Dans le domaine de l'imagerie de synthèse, Edmond Couchot évoque cette « logique de la simulation numérique » lorsqu'il propose que

Cette recréation du réel n'est évidemment qu'approchée, mais la logique de la simulation tend à l'accomplir avec le maximum de précision, selon des lois rationnelles qui le décrivent ou l'expliquent. Elle cherche à recréer de toutes pièces une réalité autonome, avec toute sa profondeur structurelle et fonctionnelle. [...] L'espace virtuel [...] est un espace sans lieu déterminé, où toutes les dimensions, toutes les topologies, toutes les lois d'associations, de déplacements, de translations, de projections, sont possibles : un espace utopique (Couchot, 1991, pp. 15-16).

En fin de compte, selon la définition de Gilles Brougère évoquée plus haut, il apparaît que la manipulation de SFM est règlementée par trop de procédures strictes pour permettre à ce dernier de prétendre inconditionnellement au statut de jouet: tout en permettant différentes activités découlant de la manipulation du jeu original, sa fonction n'est pas aussi vague que celle de "jouer avec une figurine ou une auto miniature " (Brougère, 1992, p. 39). De plus, bien qu'elle soit beaucoup plus simple que celle d'un logiciel d'infographie 3D, l'interface contextuelle et spécialisée de SFM n'est que partiellement intuitive, et ses diverses opérations doivent se succéder dans un certain ordre pour produire un résultat tangible. Cependant, parce que ces "règles" d'utilisation débouchent ultimement sur une création mal quantifiable et plus ou moins originale, il est clair qu'une certaine liberté dans la manipulation de l'interface, combinée à une motivation de l'usager qui relève du plaisir, est ici à l'œuvre: sans être tout à fait un jouet, SFM/TF2 n'est certainement plus le jeu sur lequel il se base. Quelles sont les caractéristiques de ce dispositif qui l'assimilent à cet objet utilisé librement qu'est le jouet ?

18 À travers son parcours analytique par rapport au jeu dans le contexte du Serious Game, Gilles Brougère relève les pièges d'une typologie qui s'avérerait ultimement plus restrictive que révélatrice :

De cette analyse, je tire l'idée qu'il est vain de délimiter le jeu. [...] Il n'y a pas dans le monde des jeux et des non-jeux mais des pratiques qui sont pensées par certains comme des jeux et par d'autres comme des non-jeux. Ce que nous pouvons tenter de comprendre, c'est pourquoi telle pratique est pensée comme jeu et dans le même temps pourquoi il peut y avoir ambiguité (2012, p.122).

19 Suivant sa suggestion, plutôt que de délimiter le jeu (et par extension le jouet) en tant que concept, on tentera de définir le binôme SFM/TF2 selon les contextes et les procédés particuliers qui y sont associés. Pour les besoins de notre exposé, on déclinera ces agissements selon deux principales catégories d'utilisateurs : les joueurs de jeux vidéo possédant une expérience préalable du jeu original, et les réalisateurs de cinéma cherchant à prévisualiser au moyen d'outils numériques la création de leur film à venir. Les agissements des premiers relèvent en partie du machinima, alors que ceux des seconds seront considérés comme des activités émergentes.

\section{Le joueur et le jeu de la fiction}

C'est par l'entremise de son portail Internet que Valve a initialement offert SFM comme instrument gratuit de création numérique à ses abonnés. En considérant que la manipulation de l'interface de SFM implique une familiarité avec celle du jeu vidéo qui lui 
est sous-jacente, le caractère ciblé de cette mise en disponibilité est indiscutable: on ne s'adressait pas ici à une communauté artistique au sens large (ou cinématographique en particulier), mais plutôt à une collectivité de joueurs accoutumés au jeu qu'est TF2. En plus de l'outil lui-même, c'est le cadre diégétique, voire la mythologie de cet univers imaginaire, qui était ainsi offert en premier lieu à l'usager comme matière à récit.

Or cette mythologie est riche, et se prête bien à la fabrication de récits additionnels : la base du jeu existant propose un conflit entre deux camps adverses dont le caractère sportif (les couleurs des uniformes sont les seules véritables différences entre les belligérants) débouche facilement sur une approche narrative à caractère épisodique. $\mathrm{Du}$ côté des personnages, les différents avatars expriment par leur apparence et leurs comportements des personnalités claires, ou du moins répondant aux archétypes d'un certain genre cinématographique, celui du film d'action ou d'aventure. Enfin, des bandes dessinées créées par les concepteurs du jeu viennent développer l'arrière-plan historique de l'univers diégétique du jeu. À ces récits dessinés s'ajoutent des courts métrages produits par les concepteurs du jeu et mettant en scène les principaux personnages de TF2 : ce sont ces films, regroupés sous la série Meet The Team et diffusés en ligne, qui servent de première référence aux apprentis réalisateurs se familiarisant avec SFM.

D'une manière plus concrète, la variété des modèles infographiques disponibles influence les premières initiatives narratives réalisées dans l'univers du jeu. Les modèles [assets] disponibles par défaut dans TF2 (personnages, accessoires, décors et environnements polygonaux) vont guider le joueur-réalisateur dans la mise en scène de ses premiers récits réalisés dans SFM. Le caractère limité de cette palette d'éléments n'est pas à priori une mauvaise chose, puisqu'il permet au départ de restreindre les possibilités de mise en scène, évitant ainsi à un néophyte de la réalisation filmique de tomber dans certains pièges connus (par exemple un projet trop ambitieux qui demeurera une œuvre inachevée).

Mythologie et ressources de modélisation : ce double héritage débouche en premier lieu sur des intrigues non soumises aux objectifs et aux règles du jeu original, mais qui constituent des permutations spécifiques des différents éléments diégétiques déjà disponibles. À la lumière de différents exemples de films produits par les adeptes de SFM, on remarque dans leur évolution que ceux-ci tendent à gagner en originalité : le ton humoristique propre à TF2 suggère la satire des récits d'aventures popularisés par les blockbusters américains, et ce sont les clichés du cinéma de genre qui deviennent alors une invitation à la créativité, de la même manière qu'ils peuvent, dans un film interactif, faciliter la participation du joueur selon des stratégies bien connues de l'amateur de ce type de récit (Perron, 2003, p. 238). Éventuellement, les parodies de TF2 (comme Intelligent Heavy Educates Spyper About an Important Game Mechanic de Minifett, 2011) laissent ainsi la place à des récits " sérieux » ou dramatiques, à contre-courant du ton habituel du jeu vidéo original (Cinematic TF2, Merchante, 2012), récits qui dans des cas moins fréquents incluront un remaniement d'une partie de l'univers diégétique du jeu (Dome (the demo), Fayette, 2012). Ces dernières œuvres impliquent la fabrication ou l'importation de modèles originaux dans l'interface existante, ce qui reflète une appropriation grandissante du dispositif par ses utilisateurs qui pourront notamment choisir de délaisser l'esthétique Cartoon en faveur d'un style visuel plus personnel.

En transformant l'espace ludique en plateau de tournage virtuel, cette approche par laquelle le joueur devient réalisateur s'apparente à celle des créateurs de machinima, dont les courts métrages fabriqués à l'aide de jeux vidéo comme Quake (Id Software, 1996), 
The Sims (Maxis, 2000) et Halo (Bungie, 2001) font aujourd'hui l'objet de festivals en ligne et de plusieurs ouvrages théoriques (Picard, 2007). Cependant, contrairement à une époque où les technologies disponibles limitaient l'enregistrement d'un récit à une performance ponctuelle qui devait être renouvelée autant de fois que nécessaire, SFM/ TF2 permet facilement de créer ou modifier les éléments diégétiques d'une prise existante en la faisant se dérouler de manière identique autant de fois que désiré. Le "tournage " est ainsi non plus simulé, mais bien déconstruit dans le temps, alors que le joueur ne devient plus l'artiste de performance (performer) suggéré par Henry Lowood face au machinima ${ }^{1}$, mais se transforme plutôt en un créateur de films, cumulant tour à tour tel un homme-orchestre les différents métiers d'un plateau de tournage afin de construire des récits par itérations successives.

Cette déconstruction est clairement représentée dans la conception de l'interface de SFM où, malgré la ressemblance avec l'interface d'un logiciel de montage, l'usager ne manipule plus des images filmées dans le temps, mais bien des actions [events] enregistrées dans l'espace. En plus d'assurer une grande liberté créative au joueurdevenu-réalisateur, cette façon de faire demeure suffisamment conviviale pour éviter une préparation trop laborieuse telle que celle propre au «machinima de performance » et impliquant la participation de plusieurs intervenants ainsi que la conception d'un scénarimage (Johnson et Pettit, 2012, p.71). De manière plus subtile, il est intéressant de considérer les implications d'un processus de création filmique où la présence de multiples séries de cadres n'est plus un prérequis au montage, mais bien la dernière action d'un réalisateur désirant "figer » son récit, une fixation discrétionnaire et ponctuelle : la liberté créative dont jouit le metteur en scène rappelle ainsi celle de l'écrivain qui, jusqu'à l'ultime remise du manuscrit à l'imprimeur, dispose encore de l'entièreté des permutations de la langue pour effectuer une modification à son texte.

En résumé, qu'il s'agisse de jouer à TF2 ou de créer un film grâce à SFM, le joueur devenu « joueur-réalisateur » explore les différents potentiels d'un double dispositif, que ce soit au départ celui de disputer une joute compétitive avec des adversaires ou par la suite de créer un récit filmique à plus ou moins forte parenté diégétique avec l'univers du jeu. Du premier contexte vers le second, les buts et règles du jeu original s'assouplissent ou disparaissent, alors que les moyens disponibles gagnent en efficacité : le joueur passe ainsi d'une activité sévèrement balisée à une nouvelle activité qui apparaît plus libre et moins quantifiable ${ }^{2}$. Toutefois, comme dans le jeu original, ce joueur se soumet volontairement à certaines règles afin de créer ses films (par exemple l'utilisation d'un cadrage rectangulaire). De plus, il limite essentiellement ses explorations à l'univers diégétique de TF2, ce qui selon Brougère est également une caractéristique propre à l'interaction de l'enfant avec le jouet :

Le rôle de la représentation dans le jouet est de susciter l'envie de pénétrer dans un univers particulier, qu'il soit réaliste ou imaginaire. Pour cela il doit être désirable, exaltant, à tout le moins évocateur. C'est ainsi que le jouet ne propose pas à l'enfant un double de la réalité, un accès au monde adulte, mais un support d'action ou de passions ludiques (Brougère, 2003, p.81).

Cette portée des actions du joueur circonscrite à l'espace virtuel du jeu est différente de celle des pratiques de prévisualisation du réalisateur, pratiques qui par définition influenceront les tâches de création filmique prenant place subséquemment lors du tournage en milieu réel. 


\section{Le réalisateur et la création d'un pré-film}

Bien que les responsables des superproductions hollywoodiennes continuent aujourd'hui à sous-traiter leurs animatiques à des entreprises spécialisées, on remarque que SFM commence à être utilisé à des fins de prévisualisation lors de la production de films de moindre envergure, par exemple le long métrage indépendant terre-neuvien On The Run du réalisateur Mark Spurrell, actuellement en phase de montage (Spurrell, 2016). Si cette tendance devait perdurer dans les années à venir, on peut envisager un nombre croissant de cinéastes fabriquant leurs propres animatiques aussi aisément qu'ils dessineraient leurs propres scénarimages. Pour l'instant, et en considérant les approches ayant cours actuellement en prévisualisation numérique, tentons de répondre à la question suivante : en faisant appel à un logiciel comme SFM pour réaliser ses animatiques, comment la démarche créative d'un tel metteur en scène se distinguerait-elle de celle du joueurréalisateur explicitée plus haut?

29 À l'instar du joueur jouant à TF2, le réalisateur est encadré par des règles dans l'accomplissement de ses activités quand il fabrique un film, particulièrement lors du tournage où de multiples imprévus peuvent l'obliger à des compromis créatifs parfois sévères. Pour anticiper ces difficultés, les outils de prévisualisation doivent valoriser une simulation fidèle de la création cinématographique afin que les différences entre les plateaux virtuel et physique demeurent minimes. L'une des forces de la prévisualisation 3D est justement d'éviter la remédiation d'une création vers une autre, comme l'obligent notamment d'autres outils tels que le scénarimage: «Le scénarimage ne peut jamais représenter avec précision les dimensions, l'échelle ou la perspective ", explique Daniel Grégoire, qui a dirigé la prévisualisation [3D] sur Star Wars, épisode II : L'Attaque des clones et Star Wars, épisode III : La Revanche des Sith. «La prévisualisation va plus loin en vous montrant tout ce qui entre en jeu dans le monde réel» (Metz, 2006, p. 3, ma traduction). Cette équivalence en est une de logistique, mais également de création, à mesure que les choix artistiques contenus dans l'animatique ne sont plus remédiés, mais essentiellement transposés dans le film final.

À cet effet, deux différences significatives entre un système de prévisualisation tel que SFM/TF2 et la réalité du tournage qu'il anticipe méritent d'être mentionnées. La première découle de l'absence d'intervenants autres que le réalisateur lui-même: tel que mentionné précédemment, parce qu'il ne peut contrôler qu'un nombre limité d'actions prenant place simultanément, le cinéaste est forcé de construire ses mises en scène en plusieurs " passes » successives, avec l'option de modifier à loisir chaque détail de la mise en scène sans mettre en péril l'ensemble de la prise. Cette procédure ne pourrait évidemment pas être dupliquée sur le véritable plateau de tournage où les différents techniciens se partageront les multiples tâches menant aux images finales, impliquant ainsi le risque de ne pouvoir tout synchroniser comme prévu lorsque la caméra filmera. Une seconde différence est qu'en construisant son pré-film dans un environnement parfaitement contrôlé, le metteur en scène réduirait de manière importante (voire complète) la part de création relevant du hasard (désignée parfois sous le terme d'«accident heureux») ou de la spontanéité prenant place sur le plateau (spontanéité émanant du réalisateur, mais également des comédiens et des techniciens dont la contribution au film est également source de création). 
iquement, c'est ainsi l'aspect individualiste de cet outil qu'est SFM qui, en confiant le plein contrôle au cinéaste, serait susceptible d'appauvrir le processus créatif propre au plateau de tournage conventionnel... Il serait intéressant pour le réalisateur de pouvoir modifier lui-même les règles du dispositif selon le type de démarche qu'il souhaite adopter, à la manière d'un gameplayer ou méta-joueur décrit par Perron (2003, p. 252). On peut imaginer par exemple une version de SFM/TF2 qui permettrait à plusieurs intervenants de participer simultanément à la construction d'un film (à l'image du caractère multijoueur du jeu original), une stratégie qui susciterait sans doute une plus grande part de spontanéité et de hasard apte à enrichir la démarche du metteur en scène par rapport à son œuvre. Au bout du compte, ces carences entre le système virtuel et le contexte réel qu'il est censé représenter seraient sujettes à l'appréciation de chaque réalisateur.

Une fois ce compromis reconnu et accepté, qu'en serait-il de l'approche de ce réalisateur face à un tel instrument de prévisualisation ? Parce que les règles et les codes de la médiation filmique lui sont connus, le metteur en scène s'y soumettrait plus rapidement que le joueur. Cependant, à la différence de ce dernier, il est probable que le réalisateur ignorerait une grande partie des objectifs, des règles et même de la mythologie du jeu original dont est issu SFM : le fait qu'il doive se familiariser avec l'interface propre à TF2 (par exemple quant à la manipulation des différents avatars) pourra alors s'avérer frustrant, puisqu'il n'est pas là pour jouer au jeu sous-jacent.

C'est seulement lorsqu'il maitrisera cette interface de manière satisfaisante que ce « réalisateur-devenu-joueur » se retrouvera réellement en situation de jeu. En ayant la possibilité de contrôler chaque paramètre de la mise en scène avec un minimum de contraintes de temps ou de moyens, il pourra à loisir expérimenter, voir improviser la manière de mettre en images la vision mentale du film qui sera éventuellement fabriqué à partir de l'animatique. Alors que certaines règles propres à SFM s'imposeront à lui (par exemple l'impossibilité de manipuler plusieurs avatars simultanément par le biais d'un unique contrôleur), d'autres règles héritées du plateau de tournage disparaîtront : il lui sera par exemple possible de construire ses mises en scène dans le désordre en enregistrant d'abord le jeu des comédiens (en autant de prises successives qu'il y aura de marionnettes à manipuler) pour ensuite placer ses caméras, un peu à la manière de James Cameron durant la production du long métrage Avatar (Waxman, 2007).

Parce que les animatiques complétées par le cinéaste ne sont pas destinées à se suffire à elles-mêmes (comme un machinima), mais représentent à ses yeux une œuvre à venir (le film véritable) et le lieu où elle sera réalisée (l'espace réel du plateau de tournage), il y a ainsi similitude entre l'approche par le réalisateur de l'ensemble SFM/TF2 et la principale caractéristique associée selon Brougère à la notion de jouet : « [le jouet] est marqué, en effet par la domination de la valeur symbolique sur la fonction, ou pour être plus fidèle à ce qu'il est, la dimension symbolique y devient la fonction principale ${ }^{3} »(1992$, p. 37).

\section{Jeu de construction et jouet à construire}

35 Ces deux démarches, celle du joueur-réalisateur filmant des récits et celle du réalisateurjoueur créant un simulacre de son film, comportent des objectifs, des règles et des contextes différents. Toutefois, elles en viennent à converger quant à la valeur du dispositif utilisé en tant que jouet: en se positionnant comme deux stratégies à la fois distinctes et organisées de manière séquentielle dans le contexte d'un type précis 
d'activité ludique, celle du jeu de construction, ces approches renvoient à la valeur symbolique du jouet proposée par Brougère :

Réaliser, concevoir un jouet c'est traduire une représentation, un monde imaginaire ou plus ou moins réel en objet. [...] L'action proposée peut ainsi prendre un sens fictif [...] avec une ouverture ludique autour de la représentation qui permet à l'enfant d'échapper à la fonction proposée. [...] On peut analyser de façon similaire le développement de Lego qui sans abandonner son principe de construction autour de la brique propose aujourd'hui des boittes thématiques autour de représentations (pirates, science-fiction, moyen-âge) transformant le jeu de construction en jouet à construire dans la mesure où la représentation prend le pas sur la fonction-construction, ou devient la fonction principale, la construction se limitant à être le préalable nécessaire au jeu (1992, p. 40, je souligne).

Cette distinction entre deux sous-types d'un même jouet a été examinée en détail dans le cas du célèbre Lego par Maaike Lauwaert qui constate au début des années 2000 un changement de paradigme dans l'évolution du célèbre ensemble de briques, notamment en ce qui a trait au potentiel narratif de ce jouet. Aux phénomènes de fiction et de représentation évoqués par Brougère pour qualifier la manipulation du jouer une fois qu'il est construit, Lauwaert y ajoute la notion de narrativité :

Concevoir et construire sont devenues des éléments de jeu moins importants, tandis que les éléments relevant du jeu de rôle, de l'action et des récits ont été poussés au premier plan. [...] En examinant les brochures Lego introduisant les nouveaux jouets Lego pendant cette période, nous voyons le discours passer des briques à l'action, de la construction à la narration, du processus au produit (Lauwaert, 2009, p. 59-60, ma traduction).

L'auteure rappelle que les ensembles Lego traditionnels ne comportaient pas de manuels d'instruction, mais plutôt des exemples en images quant à ce qui pouvait être construit au moyen des briques comprises dans la boîte. Elle oppose la flexibilité de ces ensembles " ouverts" au caractère presque figé des ensembles dits "thématiques", conçus pour être assemblés d'une manière unique, et représentant souvent une œuvre dérivée provenant « d'un autre média tels un film, une série télévisée, une bande dessinée ou un jeu vidéo » (figure 5). 
Figure $5 \mathrm{~A}$

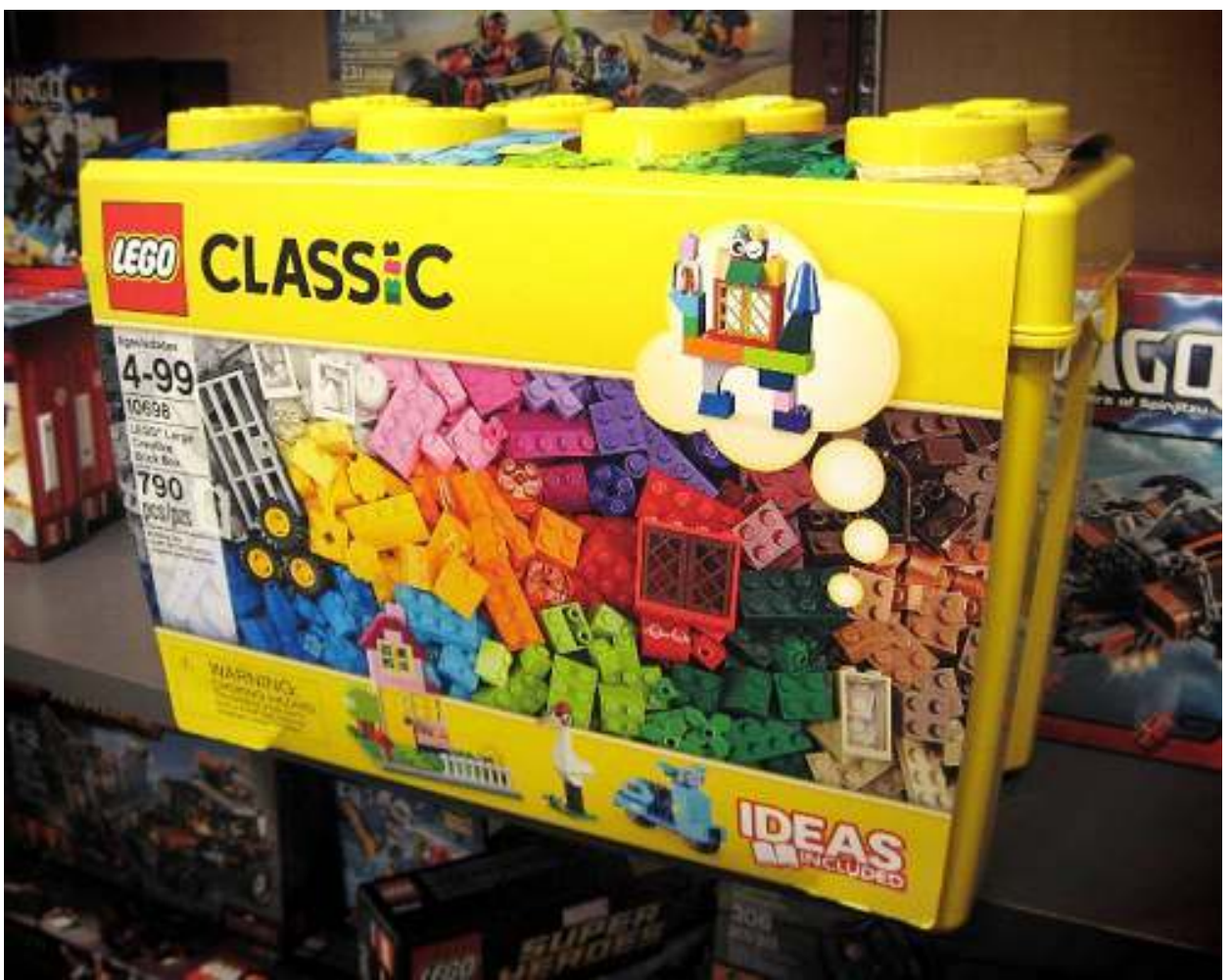

UN ENSEMBLE LEgO MODERNE DIT « OUVERT ". LES BRIQUES « gÉNÉRIQUES » PEUVENT ÊTRE COMBINÉES SELON LES gOÛTS DE L'USAgER, ALORS QUE DIFFÉRENTES SUggESTIONS D'ASSEMBLAgES APPARAISSENT SUR LA BOÎTE SOUS FORME D'IMAgES, AINSI QUE DANS UN LIVRET INCLUS À L'INTÉRIEUR. 


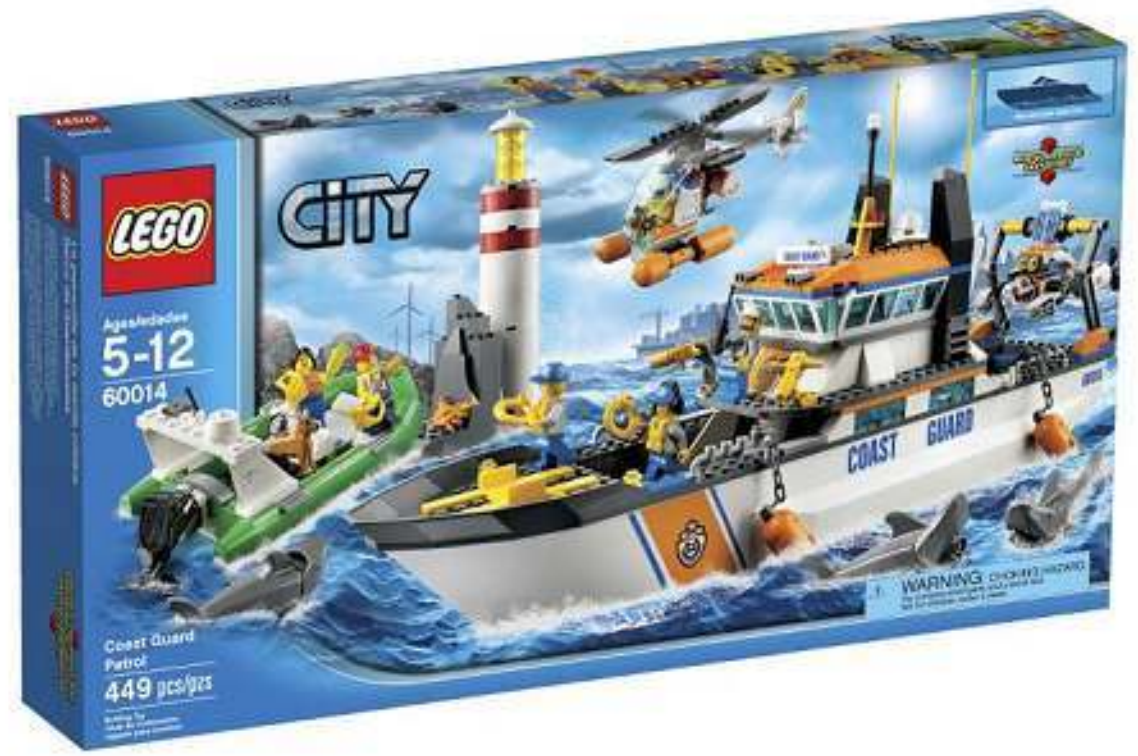

Un ensemble Lego dit « thématique » où l'on doit assembler les briques selon un concept précis, celui d'une patrouille navale. Plusieurs briques sont conçues spécialement à cette fin, telles que celles formant la coque du navire principal. Image libre de droits. fabriqués subséquemment, Lauwaert suggère ici une manière de distinguer les approches des joueurs et des réalisateurs en ce qui a trait au dispositif SFM/TF2. Selon cette analogie, on invite le joueur à se familiariser avec ledit dispositif à la manière d'un jeu de construction pour bâtir des récits filmiques. Puisque le nombre des assemblages possibles dépend en partie de la variété des éléments disponibles, le joueur a la possibilité d'enrichir l'environnement diégétique de TF2 en y ajoutant des modèles originaux, l'équivalent de «briques » qu'il concevra lui-même (ou se procurera d'un tiers). Pour guider ses assemblages, le joueur est encouragé à s'inspirer de la mythologie du jeu original, des films existants ou similaires et enfin des conventions du genre, cet ensemble de références prenant alors le rôle des différentes images sur une boîte de Lego non thématique.

À l'inverse du joueur, le réalisateur approche SFM/TF2 avec un objectif précis, celui de créer un pré-film fidèle à la vision mentale qu'il en a à ce moment-là. Une fois la première incarnation de son simulacre complétée, la recherche créative du réalisateur se concrétisera dans l'élaboration ludique de permutations subséquentes du pré-film initial : il s'agit bien ici du jouet à construire (le futur film) dont parle Brougère, dont l'image sur la boîte (le pré-film) est précise et les étapes pour y parvenir clairement identifiées, et dont « la construction se limite à être le préalable nécessaire au jeu », jeu qui pourra perdurer selon le plaisir que retire le réalisateur à transformer inlassablement son premier simulacre jusqu'à atteindre une maquette qu'il jugera « définitive » du film à réaliser. 

TF2 qui l'assimile à un jouet, celle de participer à la création de mondes fictionnels selon la théorie du "faire-semblant» [make-believe] élaborée par Kendall Walton. En considérant que les modèles utilisés dans les animatiques peuvent représenter de manière adéquate les personnages, les environnements et les différents accessoires qui prendront éventuellement part au film subséquent, on proposera que des logiciels comme SFM/TF2 relèvent de ce que Walton nomme des « supports » [props] servant à produire un certain type de fiction, tels que les poupées et les petites autos utilisées par les enfants :

Poupées et camions-jouets [...] sont conçus pour être des supports ; ils ont été fabriqués spécialement à cet effet. Telle est leur fonction, ce pour quoi ils existent [...]. En outre, ces poupées et ces camions-jouets sont destinés à être non seulement des accessoires, mais des accessoires dans des jeux d'un certain type, des jeux dans lesquels ils génèrent certaines sortes de vérités fictives : les poupées sont destinées à «compter comme» des bébés et les camions jouets comme des camions. Je qualifierai d'«autorisés » ces jeux dans lesquels un accessoire donné y remplit sa fonction. (Walton, 1990, p.51, ma traduction).

Ce premier jeu de faire-semblant, jeu où le réalisateur joue à construire une animatique par le biais d'un logiciel qui « compte comme » son plateau de tournage, précède ainsi un second jeu apparenté qui impliquera le spectateur dans la salle de cinéma, alors que le film deviendra le support par lequel ce même spectateur se projettera dans la fiction diégétique dépeinte à l'écran. Une distinction entre ces jeux relève du caractère dit " vivide » des deux types de représentations proposées, alors que le film complété sera perçu comme une invitation à la fiction plus riche et persuasive qu'une animatique 3D, et que le spectateur appréhendera cette fiction de manière plus convaincue parce que plus spontanée, comparativement à un réalisateur qui fabrique sa prévisualisation de manière délibérée à partir d'un scénario (Walton 1990, pp. 13, 297).

En accomplissant de telles démarches de création menant à une fiction déclinée du jeu original, le joueur-réalisateur participe à ce qui correspond selon Walton à un «jeu autorisé » [authorized game], c'est-à-dire que le film ainsi produit conserve une plus ou moins grande cohérence avec l'univers fictionnel de TF2 tel qu'imaginé par ses concepteurs. Le réalisateur-joueur participe lui aussi à un jeu autorisé, dans la mesure où les modèles de ses animatiques reflètent fidèlement l'univers diégétique du film à venir, du moins tel qu'il se l'imagine à ce moment-là à partir du scénario écrit, voire du scénarimage. Alors que le premier de ces usagers est initialement intéressé par les possibilités du jeu original devenu outil de création filmique, le second cherche davantage à concrétiser une vision de son œuvre qu'à apprivoiser la mythologie de TF2 : on retrouve cette dualité dans les accessoires décrits par Walton, qui postule que

[...] les supports ne sont pas toujours des outils au service du «faire-semblant». Parfois, le «faire-semblant » est un moyen pour comprendre les supports. [...] Un jeu peut être concocté tout simplement afin de clarifier ou d'exposer les caractéristiques des accessoires, tout simplement pour que nous puissions observer leur rôle dans ce jeu. Ceci est un faire-semblant au service de la connaissance des accessoires. Je qualifie ce faire-semblant comme étant orienté vers le support, et l'oppose au faire-semblant orienté vers le contenu, dont l'intérêt réside dans le contenu de l'imaginaire, dans le monde fictif (Walton, 2015, p.176, ma traduction).

Ce caractère distinctif des fictions orientées vers le statut donné au support [prop-oriented ] plutôt que vers le contenu fictionnel [content-oriented] renvoie ainsi au binôme du jeu de construction/jouet à construire. 


\section{Le contexte cinématographique actuel}

nécessaire entre l'animatique 3D et le film à venir diminue en importance, de nouvelles interrogations d'ordre logistique et artistique se font pressantes. Notons par exemple les bouleversements dans la contribution des intervenants traditionnels d'une production filmique, une contribution désormais sujette au diktat d'une animatique complétée bien avant le début du tournage, ou encore comment la première instance de l'acte créatif incarné par cette même animatique peut rendre redondante la fabrication de son film pour le réalisateur, comme le décrit Steven Spielberg:

J'ai l'occasion de faire le film avant de faire le film. [...] Le seul problème est que vous perdez environ $25 \%$ de la spontanéité à faire des découvertes sur le plateau de tournage parce que vous tombez amoureux de la prévisualisation. Vous ne laissez pas à votre imagination la chance de voler très haut quand vous vous trouvez dans le modèle 3D réel qu'est le plateau de tournage (Desowitz 2008, p. 45-46, ma traduction).

À la manière du cinéma d'animation traditionnelle où chaque dessin est présagé par le biais d'une animatique dessinée bien avant le début de la production, on peut suggérer que les animatiques réalisés aujourd'hui en infographie 3D ne représentent plus simplement une incarnation ponctuelle du scénario d'un film, mais tendent plutôt vers le simulacre à mesure qu'ils évoquent avec une fidélité grandissante un film situé en aval dont la majorité des composantes visuelles (découpage technique, direction artistique, casting des personnages, etc.) ne sont pas définies en amont. Pour reprendre la fable citée par Jean Baudrillard de la carte «si détaillée qu'elle finit par recouvrir très exactement le 
territoire ", la prévisualisation moderne devient cette nouvelle carte "qui engendre le territoire " (Baudrillard, 1981, p.15-16) : l'animatique devient lui-même un support tel que décrit par Walton, alors qu'il en vient à « compter comme » le film à venir plutôt que simplement l'anticiper, comme l'évoque le monteur Elliot Graham lorsqu'il espère que le montage final de Superman Returns (Bryan Singer, 2006) pourra «satisfaire aux attentes découlant d'une très belle prévisualisation » (Meyer Burnett, 2006, ma traduction). Cette concurrence artistique entre les deux objets concorde avec la proposition d'une "dialectique constante du média numérique avec des médias antérieurs" (Bolter et Grusin, 1999, p. 50). Si le dispositif issu du jeu se substitue ainsi au cadre traditionnel de création cinématographique, comment y situer le réalisateur ? Encore une fois, les notions d'ordre ludique peuvent nous suggérer des pistes pertinentes.

\section{Player ou Gamer ?}

En considérant le système de classification des jeux proposé par Roger Caillois (cité dans Perron, 2003, p. 240-241) et constitué d'un axe continu entre les pôles opposés de paidia et ludus, les deux permutations du même jouet décrites plus haut occuperaient des positions variées selon le point de vue adopté : au premier abord, SFM est de toute évidence un outil structuré et pragmatique, dont la fonction unique est de créer des récits filmiques à partir de ressources originales ou préexistantes, et ce en respectant les limitations de l'interface qui font ainsi office de règles. Par conséquent, cet instrument devrait tendre vers le pôle ludus de l'axe. Cependant, chacun des deux types d'usagers observés ici manipule SFM comme le prolongement d'une activité apparentée (jouer à un jeu multijoueur pour l'un, créer un film sur un plateau de tournage pour l'autre), activité dont le caractère organisé et contraignant s'avère supérieur à celui de mettre en scène des récits filmiques dans un jeu vidéo.

Que ce soit le joueur qui laisse derrière lui le caractère compétitif et quantifiable du jeu original, ou plutôt le réalisateur qui échappe au chaos organisé du plateau de tournage où chaque minute coûte cher et où les distractions sont nombreuses, ces utilisateurs sont maintenant libres de créer à leur rythme le film qui leur plait: l'idée que celui du réalisateur soit un simulacre qui servira par la suite à compléter une tâche professionnelle ne change rien au fait que ce pré-film est fabriqué de manière ludique, du moins une fois que sa première incarnation est complétée, laissant alors la place à l'exploration par l'artiste de sa propre vision créatrice. Cette valorisation du plaisir de chaque utilisateur improvisant la construction en temps réel de son récit sans se soucier de produire un résultat attendu (tous les films peuvent être différents) relève davantage de la notion de paidia que celle de ludus.

SFM peut donc être décrit comme un outil de création filmique qui s'apparente à un jeu, mais que certains utilisateurs seraient tentés de considérer comme un jouet dans la mesure où il se distingue d'une activité apparentée, et ce par l'assouplissement, la disparition ou le remplacement avantageux de ses buts, règles ou moyens disponibles. Cette description de SFM suggère qu'une qualité commune aux deux types d'utilisations décrites plus haut est une acceptation par les usagers des contraintes subsistant au sein du système, une illustration de l'attitude ludique proposée par Suits, et qui est encouragée par les concepteurs de SFM travaillant chez Valve : un exemple en est le narrateur de leurs tutoriaux vidéo qui se présente à nous comme un camarade de jeu, familier avec l'interface et la diégèse de TF2, et qui nous fait rapidement oublier que nous 
sommes en train d'apprendre le maniement d'un outil de création filmique plutôt que la navigation de base dans le cadre d'un jeu vidéo.

51 Cette attitude peut être explicitée selon la distinction suggérée par Bernard Perron entre le joueur qui accomplit une activité pour le plaisir de jouer (player) par rapport à son homologue qui se soumet volontairement à des règles définissant s'il finit par gagner ou perdre (gamer) : «Le joueur ne joue pas pour gagner ou perdre ou pour obtenir un meilleur score. Un tel résultat n'existe pas. Le joueur n'a pas à tricher pour atteindre la fin du jeu. Les joueurs peuvent être déçus, mais ils ne perdent jamais » (Perron, 2003, p. 249, ma traduction).

Par opposition, le joueur-réalisateur est un gamer lorsqu'il joue à TF2 plutôt qu'à SFM : on pourrait se demander si le réalisateur-joueur est lui aussi en quelque sorte un gamer lorsqu'il est soumis aux défis, contraintes et imprévus du plateau de tournage. Sa victoire serait de réussir envers et contre tout à compléter un film (mais peut-être pas nécessairement en créant celui qu'il désire). Quoi qu'il en soit, en acquérant une plus grande liberté et flexibilité dans leurs actions, et peut-être surtout en définissant euxmêmes leurs objectifs à atteindre, joueur et réalisateur se métamorphosent tous deux en joueurs de type player lors de leur passage vers SFM, alors que la fabrication d'un film s'apparente plus que jamais pour eux à la manipulation ludique d'un jouet.

\section{Conclusion}

Selon une perspective ludique, l'ensemble SFM/TF2 comporte des caractéristiques qui l'assimilent davantage à un jouet qu'à un jeu. Cette perspective est illustrée par l'approche initiale qu'en auront les joueurs qui ne sont plus soumis aux buts et règles du jeu original, mais également les réalisateurs de métier libérés des contraintes logistiques du plateau de tournage lorsqu'il s'agit de produire la prévisualisation 3D d'un film projeté.

Cette liberté et cette flexibilité permises par le logiciel favorisent une attitude ludique tant chez le joueur que chez le réalisateur, et par conséquent tendent à assimiler la création cinématographique à un jeu et le dispositif SFM/TF2 à un jouet, dans la mesure où subsistent néanmoins des règles encadrant la fabrication des films qui en sont issus. Plus précisément, les comportements du joueur et du réalisateur face au logiciel suggèrent une similitude entre ce dernier et le phénomène du jeu de construction / jouet à construire. Confrontés à une activité plus qualifiable que quantifiable et dont les buts deviennent subjectifs, le joueur-réalisateur et le réalisateur-joueur sont davantage guidés par leur plaisir de jouer (tels des players) que par une volonté de gagner (à l'instar des gamers).

L'évolution prochaine de ces outils de prévisualisation numérique continuera vraisemblablement à valoriser une facilité de manipulation de la part des usagers profanes, une sophistication de l'esthétique et de la précision des résultats visuels et une diversité des éléments diégétiques disponibles et des permutations qui peuvent en résulter. Ce paradigme du «Low floor, high ceiling, wide walls» (littéralement "plancher bas, haut plafond, murs espacés»), associé entre autres au jeu de Lego (Resnick, et Silverman, 2005), encourage la poursuite des questionnements soulevés dans le présent texte quant à la place et au rôle du jeu et du jouet dans les activités de l'artiste-réalisateur désirant visualiser ses œuvres à venir. 


\section{BIBLIOGRAPHIE}

BAUDRILLARD J. (1981), Simulacres et simulation, Paris, Galilée, Coll. Débats.

BELL, L. (2013), « Lucasfilm will combine video games and movies to axe post-production process ", The Inquirer, créé le 20 septembre 2013. En ligne: <http://www.theinquirer.net/ inquirer/news/2295956/lucasfilm-will-combine-video-games-and-movies-to-axe-postproduction-process $>$.

BOLTER J. D. et GRUSIN R. (1999), Remediation: Understanding New Media, Cambridge, MIT Press.

BOUZEREAU, L. (1999). Making ‘Taxi Driver’, USA, Columbia TriStar. Documentaire sur DVD, 71 minutes, Son, Couleur, vidéo (Ratio 1,33).

BROUGÈRE G. (1992), « Le jouet, objet extrême », Design Recherche, no 2, septembre, pp. 37-44.

BROUGÈRE G. (2003), Jouets et compagnie, Stock, Paris, 2003.

BROUGÈRE G. (2012), « Le jeu peut-il être sérieux ? Revisiter Jouer/Apprendre en temps de serious game ». Australian Journal of French Studies, Vol XLIX, N², pp. 117-129.

COUCHOT E. (1991), « Un fracassant Big Bang ». Cinémas : revue d'études cinématographiques / Cinémas: Journal of Film Studies, Université de Montréal, Département d'histoire de l'art, vol. 1, n 3, pp. 7-20.

DESOWITZ B. (2008), « Summer Previs to the Rescue », Animation World Network, créé le 30 mai 2008. En ligne: <http://www.awn.com/vfxworld/summer-previs-rescue>.

DRISCOLL K. et DIAZ J. (2009), « Endless loop: A brief history of chiptunes ». Transformative Works and Cultures, no. 2. En ligne : <http://dx.doi.org/10.3983/twc.2009.0096>.

FRASCA G. (1999), « Ludology Meets Narratology. Similitude and Differences between (Video)games and Narrative ». Publié à l'origine en finlandais dans Parnasso 1999: No. 3, pp. 36571. En ligne : <http://www.ludology.org/articles/ludology.htm>.

GUTTMANN A. (2006 (1978)), Du rituel au record, la nature des sports modernes, Paris, L'Harmattan, coll. "Espaces et temps du sport".

JOHNSON P. et PETTIT D. (2012), Machinima : the art and practice of virtual filmmaking, Jefferson (N.C.), McFarland \& Company.

LAUWAERT M. (2009), The Place of Play: Toys and Digital Cultures. Amsterdam, Amsterdam University Press, MediaMatters.

LOWOOD H. (2005), « Real-Time Performance : Machinima and Game Studies », The International Digital Media and Arts Association Journal, Vol. 1, No. 3, pp. 10-17.

MELANÇON B. (2011), La prévisualisation 3D comme nouveau médium créatif, mémoire de maîtrise, Chicoutimi, Université du Québec à Chicoutimi. En ligne : <http:// constellation.uqac.ca/2554/1/030288729.pdf>.

METZ C. (2006), « Hollywood Reboots », PC Magazine Digital, 23 mai 2006, Vol. 25, No.9, pp. 62-74. En ligne : <http://www.pcmag.com/article2/0,2817,1954708,00.asp>. 
MEYER BURNETT, Robert (2006), Bryan's blog \#20: Love Previz, Australie, Warner Brothers. Documentaire vidéo. 8 minutes, Son, Couleur, vidéo (Ratio 1,33). En ligne : <http:// www2.warnerbros.com/supermanreturns/videoblog/>.

PERRON B. (2003), « From Gamers to Players and Gameplayers: the Example of Interactive Movies ", The Video Game Theory Reader, sous la direction de B. Perron et Mark J.P. Wolf, New York, Routledge, pp. 237-258.

PEETERS, B., FATON, J. et DE PIERPONT, P. (1992), Storyboard ; le cinéma dessiné, Crisnée, éditions Yellow Now.

PICARD M. (2007), « Machinima: Video Game As An Art Form? », Loading...: Journal of the Canadian Game Studies Association, Vol. 1, No. 1.

POWERS, J. P. (2011), « Darkness on the Edge of Town: Film Meets Digital in Phil Solomon's In Memoriam (Mark LaPore) », October no. 137, été 2011, pp. 84-106

RESNICK M. et SILVERMAN B. (2005), « Some reflections on designing construction kits for kids ». Proceedings of the 2005 conference on Interaction design and children (IDC '05), ACM, New York, NY, USA, pp. 117-122.

RUBIN P. (2016), « Magic Leap's Next Move? Bringing C-3PO to Your House », Wired, 16 juin 2016. En ligne : < http://www.wired.com/2016/06/magic-leap-lucasfilm>.

SARTO D. (2012), « An Exploration of 'Prometheus' Previs », Animation World Network, 2 août 2012. En ligne : < http://www.awn.com/vfxworld/exploration-prometheus-previs >.

SARTO D. (2015), « The Challenging Previs of 'Spectre'», Animation World Network, 10 décembre 2015, en ligne : < http://www.awn.com/vfxworld/challenging-previs-spectre>.

SPURRELL M. (2016), « Matt Workman on 3D Previsualization », entrevue (enregistrement sonore), en ligne : < http://www.thebadalwayscatchesup.com/blogu/2016/1/14/interview-withmatt-workman-cinematographer-founder-of-cinematography-database>.

SUITS B. (1990), «Construction of a definition », The Game Design Reader. A Rules of Play Anthology, sous la direction de Katie Salem et Eric Zimmerman, Cambridge, MA: MIT Press, 2006, pp. 172-191.

WALTON K. (1990), Mimesis as Make-Believe: On the Foundations of the Representational Arts, Cambridge, Mass., Harvard University Press.

WALTON K. (2015), In other shoes: music, metaphor, empathy, existence, New York, NY, Oxford University Press.

WAXMAN S. (2007), « Computers Join Actors in Hybrids On Screen », The New York Times, 9 janvier 2007. En ligne : <http://www.nytimes.com/2007/01/09/movies/09came.html>.

WOLF M.J.P. (2014), LEGO Studies: Examining the Building Blocks of a Transmedial Phenomenon, New York (NY), Routledge.

\section{NOTES}

1. En décrivant l'atmosphère de compétitivité qui entourait la pratique du célèbre jeu vidéo Doom au milieu des années 1990, Lowood explique que « Lorsque des individus et des équipes régulières de joueurs se réunissaient en clans, il s'agissait d'un moyen d'établir des réputations collectives fondées sur une grande maîtrise du jeu. Les films réalisés dans ce contexte visaient à exhiber 
leurs exploits aux autres joueurs. [...] Le résultat ne fut rien de moins que la métamorphose du joueur en artiste de performance » (Lowood, 2005, p. 13, ma traduction).

2. En abandonnant les buts et règles de TF2, SFM conserve certaines des restrictions du jeu, par exemple la manipulation des avatars dans l'espace: il est par exemple impossible pour un personnage de traverser les murs présents dans l'univers du jeu, bien qu'il soit possible pour des usagers initiés d'inclure des modifications (MODS) au code de l'engin de jeu afin de contourner nombre de ces règles.

3. Cette valeur symbolique est exacerbée davantage encore si l'on se rappelle que, malgré ses similitudes avec un logiciel de montage, SFM vise à manipuler des actions diégétiques, et non des images.

\section{RÉSUMÉS}

Par le détournement d'applications et de dispositifs associés à la pratique du jeu vidéo, un réalisateur peut dorénavant élaborer à l'avance un brouillon visuel de son film à venir. À travers l'examen de l'un de ces outils composé d'un logiciel (Source Filmmaker) et d'un jeu multijoueur ( Team Fortress 2), ce texte propose de considérer ce nouvel instrument de création filmique non plus comme un jeu, mais plutôt en tant que jouet. Cette démarche impliquera certaines passerelles possibles entre l'usage de la prévisualisation numérique impliquant l'infographie 3D, et des concepts d'ordre ludique tels que les notions de jeu de construction et de jouet à construire, ou encore la distinction entre player et gamer. En assimilant le cheminement traditionnel de création cinématographique à une pratique ludique, le présent texte suggère de mieux comprendre comment le nouveau paradigme de la prévisualisation 3D transforme désormais le processus créatif menant à la réalisation d'un film.

By diverting applications and devices associated with the practice of video games, a film director can now prepare in advance a visual draft of his upcoming film. Through the examination of one of these tools consisting of software (Source Filmmaker) and a multiplayer game (Team Fortress 2), this text proposes to consider this new instrument of cinematic creation not as a game, but rather as a toy. This approach will entail possible bridges between the use of digital previsualization involving 3D computer graphics and game theory concepts such as construction toys and model building, or the distinction between player and gamer. By assimilating the traditional process of filmmaking as a play-related activity, the text suggests a better understanding of how the new paradigm of 3D previsualization now transforms the creative process leading to the making of a film.

\section{INDEX}

Mots-clés : prévisualisation, scénarimage, animatique, réalisateur, jeu de construction, jeu vidéo, joueur, gamer, faire-semblant

Keywords : previsualization, storyboard, previs, director, construction toy, video game, player, make-believe 
AUTEUR

BENOIT MELANÇON

Centre NAD / UQAC 